\title{
Temperature dependence of singlet oxygen generation by different photosensitizers
}

\author{
Aleksander Starukhin ${ }^{1, *}$, Aleksander Gorski ${ }^{2}$, and Jacek Dobkowski ${ }^{2}$ \\ ${ }^{1}$ B.I. Stepanov Institute of Physics, National Academy of Sciences of Belarus, 220072 Minsk, Belarus \\ ${ }^{2}$ Institute of Physical Chemistry, Polish Academy of Sciences, 01-224 Warsaw, Poland
}

\begin{abstract}
The temperature dependences of the lifetimes and the efficiency of singlet oxygen generation for a set of compounds (porphyrins, metalloporphyrins, porphycene, terrylene etc.) has been measured and characterized. Based on the character of the temperature dependence of singlet oxygen generation for metalloporphyrins and porphycene it was assumed a possibility of creation a complex between organic moieties and molecular oxygen. Singlet oxygen generation of terrylene with the quantum yield of $22 \%$ was recorded in toluene at ambient temperature.
\end{abstract}

Oxygen plays a significant role in environmental chemistry, in industry, medicine, and agriculture. It is well known that organic compounds in solutions in the presence of oxygen can intensively degrade at light irradiation. One of the most significant reasons of degradation of organic compounds in solution is due to singlet oxygen generation and attendant reactions [1,2]. Singlet oxygen $\left({ }^{1} \mathrm{O}_{2}\right)$ is an energetically excited state of molecular oxygen, which is usually located in the ground triplet electronic state. The singlet oxygen may be generated as a result of a photochemical reaction between organic molecule in solution and triplet molecular oxygen. After absorption of a quantum of light, the molecule of photosensitizer $(\mathrm{P})$ is excited in the first excited singlet state $\mathrm{P}\left(\mathrm{S}_{1}\right)$, and after intersystem crossing transfer to the triplet state $\mathrm{P}$ (T1). The singlet oxygen generation proceeds with the creation of a complex $\left(\mathrm{P}-\mathrm{O}_{2}\right)$ with a weak bond (intermolecular energy transfer process) between the photosensitizer and oxygen. Upon interaction of a photosensitizer in the triplet state $\mathrm{P}\left(\mathrm{T}_{1}\right)$ with molecular oxygen in the triplet state $\left({ }^{3} \mathrm{O}_{2}\right)$, singlet oxygen $\left({ }^{1} \mathrm{O}_{2}\right)$ is formed as a result of triplet-triplet annihilation, where the photosensitizer returns into the ground electronic state. The process is controlled by process of diffusion of triplet molecules (photosensitizer and triplet oxygen molecule). Therefore, singlet oxygen is practically not generated at low temperatures, where diffusion processes are very slow or absent. This report presents the study of the temperature dependence of the generation of singlet oxygen by various photosensitizers.

The efficiency of singlet oxygen generation as a function of temperature has been measured and the results for two selected compounds are presented on Figure 1.

${ }^{1}$ Corresponding author: $\underline{\text { starukhin@ mail.ru }}$ 


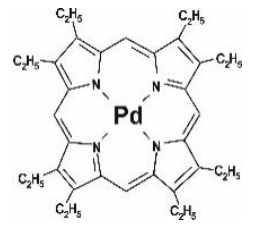

a

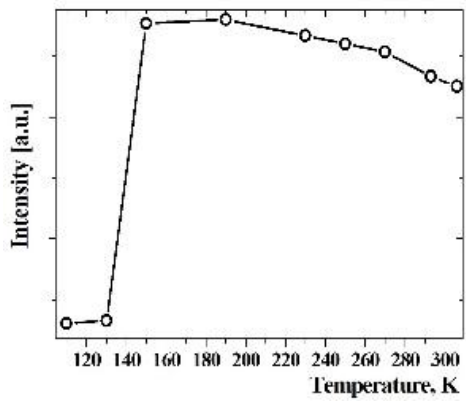

c

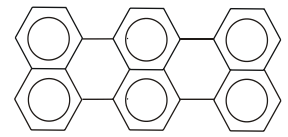

b

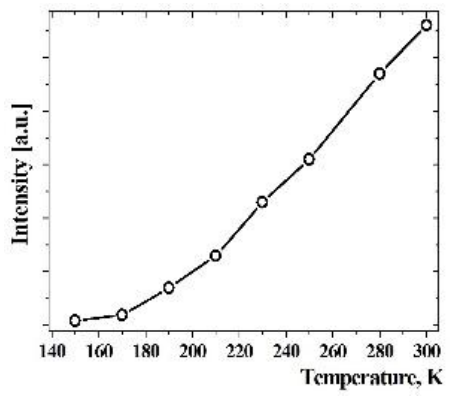

d

Fig. 1. Chemical structures of the studied compounds: a - Pd - 2,3,7,8,12,13,17,18 octaethylporphyrin (Pd-OEP); b - terrylene (TER), as well as the temperature dependence of the efficiency of singlet oxygen generation for Pd-OEP ( c) and TER (d) in toluene.

The presented results exhibit different behaviour of the efficiency of singlet oxygen generation as a function of temperature for Pd-OEP and TER in temperatures range from 170 to $300 \mathrm{~K}$. The quantum yield of singlet oxygen generation of TER were carried out and this value at $300 \mathrm{~K}$ was $22 \%$. In case of TER the efficiency of singlet oxygen generation almost linearly decreased with temperature. This fact is correlated with temperature dependence of diffusion coefficient of oxygen in solution. While, Pd-OEP is characterized by a quite weak temperature dependence of singlet oxygen generation up to $150 \mathrm{~K}$ (similar data were recorded for porphyrins, metalloporphyrins and porphycene). In the temperature range from 300 to $150 \mathrm{~K}$, even a slight increasing of the generation of singlet oxygen occurs (see Fig.1c). The generation of singlet oxygen sharply disappears at temperature of toluene crystallization.

Additionally, the dependences of phosphorescence lifetime of Pd-OEP has been measured at atmospheric pressure of oxygen in solution. In temperature range between 300 - $170 \mathrm{~K}$ the lifetime of the phosphorescence of Pd-OEP remains below $1 \mu \mathrm{s}$. At low temperature, below $170 \mathrm{~K}$ the value of the lifetime is drastically increasing in more than 7000 times up to $1,4 \mathrm{~ms}$. This results with the fact that Pd-OEP is characterized by almost $100 \%$ quantum yield of the triplet state formation (a strong spin-orbit coupling) indicates the weak influence of oxygen diffusion on the deactivation of the triplet state of Pd-OEP. The data allow to propose, that the Pd-OEP-O $\mathrm{O}_{2}$ ground state complex (which is true for several other porphyrin derivatives and porphycen) is formed in solutions.

This work was supported by the Belarus-Poland Grant № F18PLSHG-003.

\section{References}

1. A. Krasnovsky jr., Biofizika USSR 21, 748 (1976)

2. Cl. Schweitzer et al., Chem. Rev. 103, 1685 (2003)

3. D. Asturiol et al., The J. of Chem. Phys.. 139, 074307 (2013)

4. F. Rusydi et al., J. of the Phys. Soc. of Japan. 81, 124301 (2012) 